\title{
Visible-wavelength super-refraction in photonic crystal superprisms
}

\author{
J. J. Baumberg ${ }^{a), b), c)}$ and N. M. B. Perney ${ }^{a)}$ \\ School of Physics and Astronomy, University of Southampton, Southampton SO17 1BJ, United Kingdom \\ M. C. Netti, M. D. C. Charlton, ${ }^{\text {b) }}$ M. Zoorob, and G. J. Parker ${ }^{\text {b) }}$ \\ Mesophotonics Limited, 2 Venture Road, Chilworth Science Park, SO16 7NP, United States
}

(Received 2 February 2004; accepted 18 May 2004)

\begin{abstract}
We demonstrate the fabrication of superprism devices in photonic crystal waveguides with excellent transmission through 600 rows of $160 \mathrm{~nm}$ diameter holes. Broadband spectral and angular measurements allow mapping of the chromatic refractivity. This shows the ability of such devices to super-refract by more than $1 \% / \mathrm{nm}$ close to the principal band gaps, $10 \times$ more than equivalent gratings, and $100 \times$ more than equivalent prisms. Simple theories based on plane-wave models give excellent agreement with these results. (0) 2004 American Institute of Physics.
\end{abstract}

[DOI: 10.1063/1.1772521]

Photonic crystal (PC) waveguides promise many ways to miniaturize and integrate photonic devices on high functionality optical chips. An extremely wide range of components can benefit from the competing interplay between multiple diffraction and interference in such periodically patterned nanostructures. This results in many enhanced optical properties around the critical band gaps in the photonic bandstructure which can break conventional optical "design rules" such as multirefringence, ${ }^{1}$ slow light, ${ }^{2-4}$ enhanced nonlinear conversion, ${ }^{5}$ and superprisms. ${ }^{6-9}$ While considerable theoretical effort has been developed in the past few years to raise the prospect of enhanced performance, ${ }^{10,11}$ very few devices have actually been realized, spectrally tested and robustly compared to these theories. In particular, the role of the photonic band gaps in relation to the superprism phenomena have not been highlighted.

In this letter, we show state-of-the-art fabrication of PCs in the visible optical region based on a silicon platform. Planar waveguides are deposited and subsequently patterned with a range of periodic arrays of holes in different geometries, and up to 600 rows of holes across. Broadband optical characterization on a range of such devices allows us to clearly identify their excellent transmission properties, exhibiting strong band gaps with high transmission in the intervening spectral regions. A chromatic refraction technique combining angular scans with transmission spectroscopy allows us to map the super-refraction properties. Our results compare well with a simple two-dimensional (2D) plane wave theory, thus enabling further optimization of the devices.

The devices are fabricated in a standard silicon microfabrication process line, compatible with very large-scale integrated electronics, on $n$-type silicon wafers. They are based on our previous designs ${ }^{1,2,12}$ with silicon nitride waveguides $\left(\mathrm{Si}_{3} \mathrm{~N}_{4} 250 \mathrm{~nm}\right.$ nominal thickness, $\left.n=2.02\right)$ embedded in silicon dioxide $\left(\mathrm{SiO}_{2}\right)$ substrate and cladding layers $(1.7 \mu \mathrm{m}$ and $75 \mathrm{~nm}$ thick, respectively, $n=1.46$ ). The PC sections are defined using electron-beam lithography and subsequent dry

\footnotetext{
${ }^{a)}$ Also at: Mesophotonics Ltd., 2 Venture Rd., Chilworth Science Park, SO16 7NP, UK.

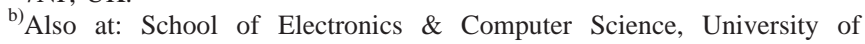
Southampton, Southampton SO17 1BJ, UK.

${ }^{c)}$ Electronic mail: j.j.baumberg@soton.ac.uk
}

etching to give large aspect ratio holes through the core waveguide layers. In this letter, we will concentrate on a particular set of devices with hole diameter $d=160 \mathrm{~nm}$, in a rectangular array of periodicity with $a=310 \mathrm{~nm}, b=465 \mathrm{~nm}$ (aspect ratio 1:1.5), with 600 rows of holes giving a total device length of $186 \mu \mathrm{m}$ [Fig. 1(a)]. However, we have examined a whole series of devices with pitches from $260 \mathrm{~nm}$ to $410 \mathrm{~nm}$, hole diameters from $160 \mathrm{~nm}$ to $360 \mathrm{~nm}$, from 10 to 1000 rows, with triangular, square, and rectangular lattices which show comparable properties to those here (to be reported elsewhere). By cleaving through notched arrays of these photonic chips, highquality end facets are obtained suitable for endfire coupling into the slab waveguides. These devices are designed to provide photonic band gaps in the visible region of the spectrum, except for the lowest band gap which is in the infrared
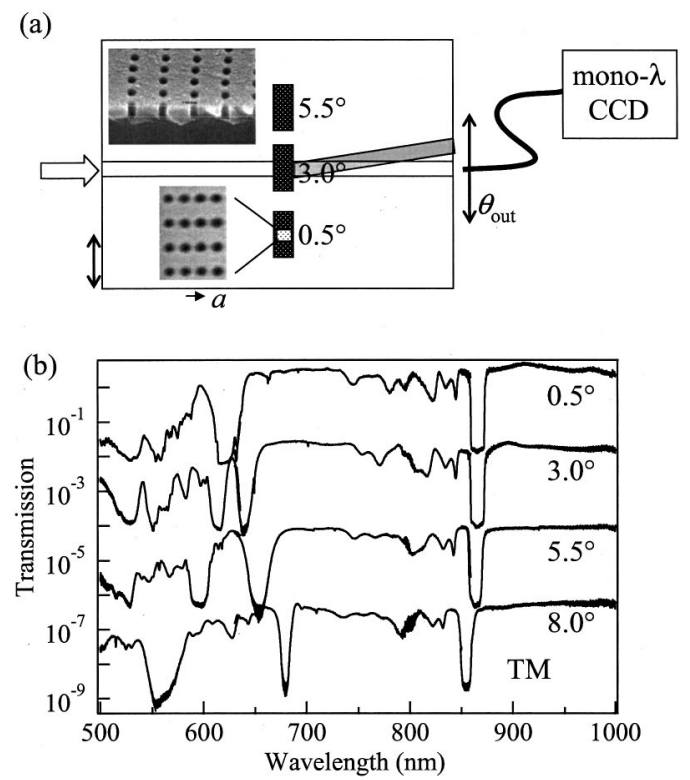

FIG. 1. (a) Design of planar PC waveguide chips containing several rectangular PC regions $(500 \mu \mathrm{m} \times 186 \mu \mathrm{m})$, together with chromatic refraction detection geometry. Insets shows scanning electron micrographs of the rectangular lattice in plan and cross section. (b) Normalized TM-polarized transmission spectra of four devices with $a$ direction successively rotated away from the propagation direction by $0.5^{\circ}-8^{\circ}$, on a $\log$ scale and shifted vertically for clarity. 
and of less interest for superprism operation here. ${ }^{7}$

In order to characterize the transmission properties of these PC waveguides, we exploit our previously developed ultrabroadband white light spectroscopy. ${ }^{12}$ This allows us to rapidly measure quantitative transmission characteristics from $450 \mathrm{~nm}-950 \mathrm{~nm}$ (and across the infrared with suitable modifications to deal with chromatic aberrations). The ability to focus such a high-brightness white source into the $300 \mathrm{~nm}$ high fundamental waveguide mode allows us to easily track different colors of light travelling in different directions inside the photonic circuit slab. Typical transverse magnetic (TM) polarized waveguide transmission spectra are shown in Fig. 1(b) for four PC structures whose lattice is successively rotated so that light propagates from $0.5^{\circ}$ to $8^{\circ}$ away from the $a$ direction of closest hole separation. The first, second, and third band gaps are clearly visible, with an extinction ratio exceeding 100:1 (limited in this experiment by transverse electric polarized stray light). Transmission is seen to be nearly $100 \%$ around the lowest band gap not just on the long wavelength (dielectric) side of the band gap but also on the short-wavelength (air) side which is prone to suffering loss from upscattering out of the waveguide. In the current context, it is extremely important to collimate the beam of light impinging on the PC to prevent averaging over different directions within the superprism. We use cylindrically compensated focusing optics to launch a $100 \mu \mathrm{m}$ wide beam at the $300 \mu \mathrm{m}$ long PC structures which are embedded at the center of $2 \mathrm{~cm}$ long waveguides [Fig. 1(a)]. A number of similar devices are fabricated on each photonic chip, each of an identical design except for a $2.5^{\circ}$ rotation in the orientation of the rectangular lattice to the propagation direction, (while the edges of the PC regions are always normal to the input facets). In between the devices are sections of an unpatterned planar waveguide that are used for calibration purposes. Thus, these bulk PC waveguides are ideal for the quantitative observations of superprism effects.

To explore the super-refraction of light at wavelengths close to the different order band gaps, we measure the angular emission of light from the PC by scanning a collection fiber along the output facet of the waveguide (Fig. 2). Due to our high brightness source, this allows rapid acquisition of spectra at each emission angle, and hence an angular-spectral map can be built up for each device under automated control. We use a multimode collection fiber coupled to an Ocean Optics HR2000 spectrometer covering the entire visible range, together with spectral filters to flatten the white light spectrum. With $10 \mu \mathrm{m}$ slits, we approach a $0.3 \mathrm{~nm}$ spectral resolution and a $0.3^{\circ}$ angular resolution (corrected for external refraction at the waveguide facet). Great care is taken to ensure that the multimode fiber tracks the exit facet of the waveguide slab as it is scanned along the chip. Such simultaneous angular and spectral characterization in waveguide geometries are crucial for identifying and mapping the chromatic-refraction performance of photonic crystal devices.

Typical chromatic-refraction maps for four devices at $0.5^{\circ}, 3^{\circ}, 5.5^{\circ}$, and $8^{\circ}$ to the $a$-direction are shown in Fig. 2. Refraction angles up to $16^{\circ}$ are observed, with clear refractive features of different signs around the different photonic band gaps. We note that such properties cannot be found in any conventional waveguide optical components. Even more significant is the large angular dispersion, up to $1 \% / \mathrm{nm}$ around these band gaps. This should be compared to

Downloaded 07 Mar 2005 to 152.78.67.79. Redistribution subject to
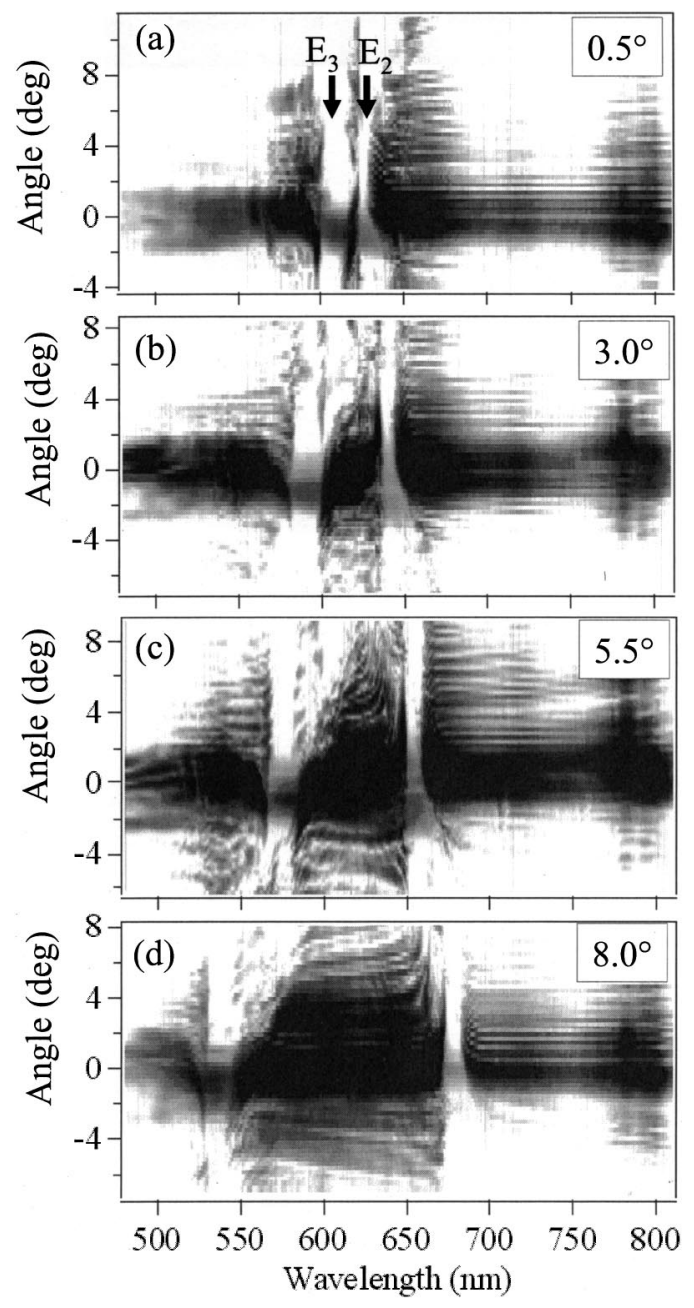

FIG. 2. Chromatic refraction maps of the broadband transmission as a function of angular deviation for each of the samples in Fig. 1(a), plotted on a $\log$ scale to easily show up the super-refraction.

$0.006^{\circ} / \mathrm{nm}$ for a conventional prism of the same effective index as the waveguide, or $0.10^{\circ} / \mathrm{nm}$ for a diffraction grating of pitch $500 \mathrm{~nm}$ embedded in the same waveguide effective index at these wavelengths. As the lattice is rotated away from the symmetry direction parallel to $a$, the magnitude of this maximum angular dispersion increases, while the band gaps shift in different directions as expected from the band diagram. This confirms that super-refractive properties are enhanced away from symmetry directions of the underlying lattice. In fact, as we show below, this is the reason we adopt rectangular PCs with a lower symmetry.

To understand the superprism effect, we adopt here a simple model based on plane wave solutions inside an infinite 2D PC of periodically modulated dielectric constant matching the effective indices of our waveguide structure. This encapsulates all of the essential physics. The band diagram for a propagation direction at an angle of $8^{\circ}$ from $a$ [Fig. 3(a)] shows the band gaps observed in our PC waveguides. Mapping the bands around the second band gap $E_{2}$ in the second [Fig. 3(b)] and third [Fig. 3(c)] Brillouin zones allows us to highlight the competition between the input propagation direction $\left(k_{i}\right)$ and the strong diffraction from lattice vector $G_{11}=\left(k_{a}, k_{b}\right)=(\pi / a, \pi / b)$. Besides producing the observed band gap, competition between interfering waves in these directions at different wavelengths produces the observed superprism effect. Light propagates AIP license or copyright, see http://apl.aip.org/apl/copyright.jsp 
(a)

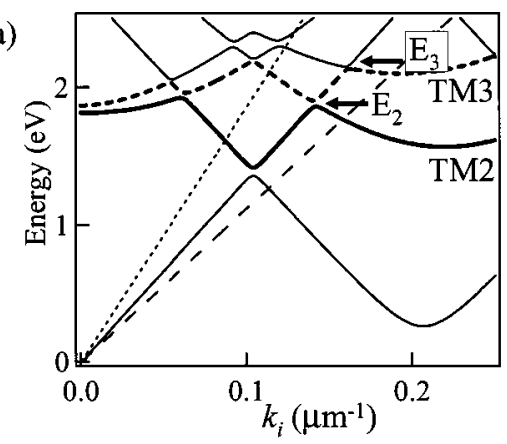

(b)

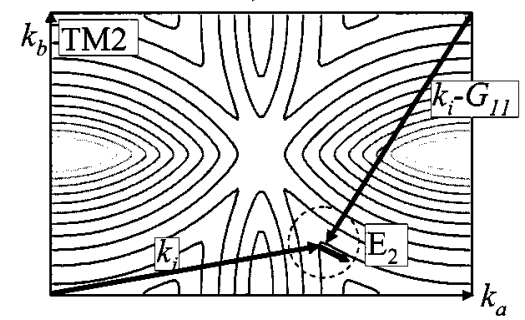

(c)

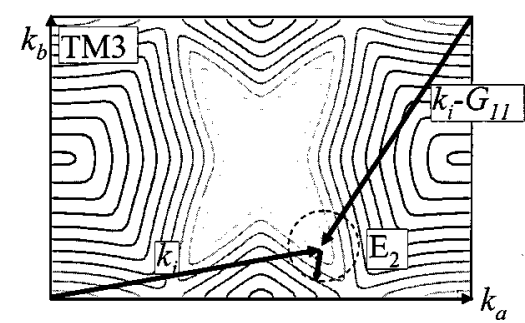

FIG. 3. (a) Band diagram along a $k_{i}$ direction which is $8^{\circ}$ from the $a$ axis showing the main band gaps observed in the waveguide mode. (b) and (c) Energy contour maps in the $\left(k_{a}, k_{b}\right)$ plane for the second and third bands, dashed circle highlights the second energy gap. The solid arrow shows the light flow direction (perpendicular to the energy contours) for the $k_{i}$ indicated (shaded arrow).

perpendicularly to the energy contours ${ }^{13}$ in Figs. 3(b) and 3(c) and, near the band gap, the transition from one dominating diffraction direction to another has the effect of separating the propagation of light of neighboring colors. On both the low- and high-energy side of the band gap, this has the effect of refracting the light away from the $a$ direction. The opposite effect occurs around the band gap $E_{3}$, with light being refracted towards the symmetry direction. Such variable control of the extent and direction of refraction is typical of PCs.

Calculating the propagation directions of light based on this plane-wave model, and accounting for the refraction through the photonic device, we arrive at predicted chromatic-refraction performance for each device (shown in Fig. 4). This provides excellent agreement with the experimental data, predicting both the sign and the magnitude of the angular dispersion. While it also predicts the shifting band gaps, as always in such plane-wave simulations which

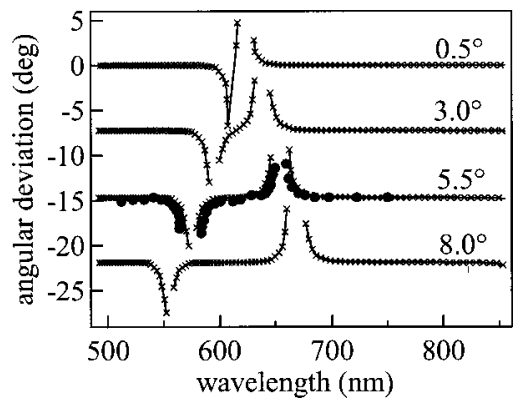

FIG. 4. Theoretical angular deviation of light at each wavelength, extracted from the plane-wave model described in the text, together with experimental data of device 3 . The curves have been shifted vertically for clarity, and scaled to match the experimental band gap positions.

fail to completely account for the three-dimensional waveguiding, the spectral position of the band gap needs to be been scaled to provide full agreement with the data. Note that as the band gap features in our transmission measurements are extremely sharp, this means that loss is not a particular issue in the device performance.

In conclusion, we have shown excellent photonic performance in the visible spectral region of PC devices built on silicon platforms. Our chromatic refraction geometry allows full automated characterization of the super-refraction of such superprism devices. The observed refraction enhancements are in agreement with plane-wave theory, and encourage development of compact integrated systems based on these components.

This work was supported by Mesophotonics Limited and EPSRC GR/N37261, GR/S62314.

${ }^{1}$ M. C. Netti, A. Harris, J. J. Baumberg, D. M. Whittaker, M. B. D. CharIton, M. E. Zoorob, and G. J. Parker, Phys. Rev. Lett. 86, 1526 (2001).

${ }^{2}$ M. C. Netti, C. Finlayson, J. J. Baumberg, M. D. B. Charlton, M. E. Zoorob, J. Wilkinson, and G. J. Parker, Appl. Phys. Lett. 81, 3927 (2002).

${ }^{3}$ S. Lan, S. Nishikawa, H. Ishikawa, and O. Wada, J. Appl. Phys. 90, 4321 (2001).

${ }^{4}$ M. Notomi, K. Yamada, A. Shinya, J. Takahashi, C. Takahasi, and I. Yokohama, Phys. Rev. Lett. 87, 253902 (2001).

${ }^{5}$ A. R. Cowan and J. F. Young, Phys. Rev. B 65, 85105 (2002).

${ }^{6}$ H. Kosaka, T. Kawashima, A. Tomita, M. Notomi, T. Tamamura, T. Sato, and S. Kawakami, Appl. Phys. Lett. 74, 1370 (1999).

${ }^{7}$ T. Baba and M. Nakamura, IEEE J. Quantum Electron. 38, 909 (2002).

${ }^{8}$ H. Kosaka, T. Kawashima, A. Tomita, M. Notomi, T. Tamamura, T. Sato, and S. Kawakami, Phys. Rev. B 58, R10096 (1998).

${ }^{9}$ A. V. Zayats, W. Dickson, I. I. Smolyaninov, and C. C. Davis, Appl. Phys. Lett. 83, 4438 (2003).

${ }^{10}$ D. Scrymgeour, N. Malkova, S. Kim, and V. Gopalan, Appl. Phys. Lett. 82, 3176 (2003).

${ }^{11}$ K. B. Chung and S. W. Hong, Appl. Phys. Lett. 81, 1549 (2002).

${ }^{12}$ M. C. Netti, M. D. B. Charlton, G. J. Parker, and J. J. Baumberg, Appl. Phys. Lett. 76, 991 (2000).

${ }^{13}$ P. St. J. Russell and T. B. Birks, in Photonic Band Gap Materials, edited by C. M. Soukoulis (Kluwer, Boston, MA, 1996), p. 71. 